\title{
ENERGIA LIVRE DA REAÇÃO DE ADSORÇÃO DE COBRE EM LATOSSOLOS ÁCRICOS ${ }^{1}$
}

\author{
Maria Lucia Azevedo Silveira ${ }^{2 *}$; Luís Reynaldo Ferracciú Alleoni ${ }^{3,6}$; José Carlos Casagrande ${ }^{4,6}$; \\ Otávio Antônio Camargo ${ }^{5,6}$ \\ ${ }^{2}$ Pós-Graduanda do Depto. de Solos e Nutrição de Plantas - ESALQ/USP. \\ ${ }^{3}$ Depto. de Solos e Nutrição de Plantas - ESALQ/USP, C.P. 9 - CEP: 13418-900 - Piracicaba, SP. \\ ${ }^{4}$ Depto. de Recursos Naturais e Proteção Ambiental - UFSCar, C.P. 153 - CEP: 13600-970 - Araras, SP. \\ ${ }^{5}$ Centro de Solos e Recursos Agroambientais - IAC, C.P. 28 - CEP: 13001-970 - Campinas, SP. \\ ${ }^{6}$ Bolsista do CNPq. \\ *e-mail: mlasilve@carpa.ciagri.usp.br
}

RESUMO: Estudou-se a energia livre da reação de adsorção de cobre em amostras superficiais $(0-0,2 \mathrm{~m})$ e subsuperficiais (na maior expressão do horizonte B) de três solos paulistas : Latossolo Roxo ácrico (LR), Latossolo variação Una ácrico (LU) e Terra Roxa Estruturada (TE), após a adição de $5,20,50,100,200,300,400$ e $600 \mathrm{mg} \mathrm{L}^{-1}$ de cobre. A adsorção de cobre pelos solos foi considerada espontânea, pois a energia livre apresentou valores negativos em todas as concentrações estudadas. Os valores de energia livre diminuíram com o aumento da dose de cobre adicionada. A TE apresentou maior energia livre de adsorção em relação aos Latossolos, sobretudo em superfície. Considerando-se o mesmo solo, os horizontes superficiais apresentaram maior energia livre para as reações de adsorção de cobre em relação aos horizontes subsuperficiais, provavelmente devido ao efeito da matéria orgânica, que apresenta alta afinidade pelo elemento.

Palavras-chave: Latossolo ácrico, cobre, adsorção, energia livre

\section{FREE ENERGY OF COPPER ADSORPTION REACTION IN ACRIC OXISOILS}

ABSTRACT: Free energy of copper adsorption was studied in surface and subsurface samples from three soils of the State of São Paulo, Brazil [Rhodic Kandiudalf (TE), Anionic "Rhodic" Acrudox (LR) and Anionic "Xanthic" Acrudox (LU)], after addition of 5, 20, 50, 100, 200, 300, 400 and $600 \mathrm{mg} \mathrm{L}^{-1}$ of copper. Copper adsorption was considered spontaneous, since the measured free energy was negative in all concentration. Free energy values decreased with the increase of added copper. TE showed higher free energy liberated than LR and LU, mainly in surface samples. For the same soils, surface horizons showed higher values for free energy liberation than subsurface horizons, probably due to the effect of organic matter, whose affinity for copper is high.

Key words: Anionic Acrudox, copper, adsorption, free energy

\section{INTRODUÇÃO}

O cobre é considerado elemento essencial à nutrição de plantas, porém quando presente em altas concentrações no solo pode apresentar caráter tóxico. O elemento pode estar presente em resíduos municipais e industriais e compostos organo-metálicos, como agroquímicos, largamente utilizados na agricultura. Ainda, fertilizantes fosfatados e corretivos agrícolas podem apresentar concentrações elevadas do metal. O uso destes componentes continuamente na agricultura pode causar o acúmulo de cobre no solo, tornando-o tóxico às plantas, aos animais e ao homem (Pezzarrossa et al., 1990).
No solo o elemento pode se encontrar complexado com a fração orgânica e a inorgânica. Diversos compostos solúveis e insolúveis são capazes de reagir com o cobre, e sua concentração na fase líquida é determinada pela solubilidade da forma menos solúvel presente no sistema.

Assim como outros metais pesados, o cobre tem sua solubilidade controlada por um conjunto de reações químicas que ocorrem na superfície dos colóides (Mattigod \& Page, 1983). Essas reações compreendem fenômenos de adsorção/dessorção, precipitação e complexação. Os óxidos de alumínio, ferro e manganês e os grupos funcionais ácidos da matéria orgânica compreendem as superfícies reativas mais

${ }^{1}$ Parte da Dissertação de Mestrado do primeiro autor apresentada à ESALQ/USP - Piracicaba, SP. 
representativas, por apresentarem as maiores superfícies específicas e, portanto, os maiores pontos de contatos com a solução do solo. Podese considerar que as reações de adsorção que ocorrem na interface sólido-líquido são um importante mecanismo controlador da atividade química de cobre não apenas na solução do solo, mas, também, em corpos de águas naturais (Petronio et al., 1996; McBride et al., 1997; Salam \& Helmke, 1998). O grau de adsorção dependerá da eletronegatividade das partículas do solo e do potencial de ionização dos íons adsorvidos. Outra propriedade conhecida é que o cobre combina com ligantes para formar complexos e quelatos estáveis, sendo, portanto, passível de formá-los com os radicais orgânicos da matéria orgânica do solo. Ainda, em valores de $\mathrm{pH}$ elevados, o cobre pode formar hidróxidos e se precipitar.

No caso de solos muito intemperizados, como os ácricos, com predominância de mineralogia oxídica na fração argila, as reações de adsorção de cobre tem características distintas de solos com mineralogia 2:1. Mesmo quando os óxidos e hidróxidos de ferro e alumínio estão presentes em baixas porcentagens no solo, os mesmos proporcionam substancial efeito na adsorção de cobre, haja vista a acentuada afinidade do metal pela superfície reativa desses componentes.

Os solos ácricos caracterizam-se pelo intenso processo de intemperismo e dessilicatização, resultando no acúmulo de óxidos de ferro (hematita e goetita) e alumínio (gibsita), além da caulinita. O caráter ácrico aparece quando o solo apresentar capacidade de troca catiônica efetiva menor que $15 \mathrm{mmolc} \mathrm{kg}^{-1}$ de argila e $\mathrm{pH}\left(\mathrm{KCl} 1 \mathrm{~mol} \mathrm{~L}^{-1}\right)$ maior ou igual a 5,0; ou $\triangle \mathrm{pH}(\mathrm{pH} \mathrm{KCl}-\mathrm{pH} \mathrm{H} \mathrm{O})$, maior ou igual a 0,1 , em uma profundidade de $125 \mathrm{~cm}$ (FAO, 1989).

Os solos são heterogêneos na sua constituição e seus atributos influenciam os fenômenos de adsorção de maneira muito complexa. É, então importante que se conheçam algumas interações do cobre com o solo, para melhor entendimento de como, quando e onde ele é retido no sistema, uma vez que esses mecanismos de retenção controlam seu destino no sistema agroecológico, sobretudo nos solos altamente intemperizados como os ácricos, nos quais informações na literatura em relação ao comportamento dos metais pesados são restritas.

A partir dos resultados de adsorção de cobre, pode-se fazer algumas inferências, como o cálculo termodinâmico da energia livre das reações $\left(\triangle \mathrm{G}_{0}\right)$ envolvidas. As mudanças na energia livre que ocorrem quando um elemento é adsorvido podem servir como medida da extensão ou a força que guia determinada reação (Singh, 1971). Seu sinal indica se a reação é espontânea ou não, em um sistema fechado, a temperatura e pressão constantes. Valores de $\triangle \mathrm{G}_{0}$ negativos indicam que a reação é exotérmica e espontânea; por sua vez valores positivos indicam que a reação é endotérmica e não pode ser espontânea. Seu valor também indica quão distante do equilíbrio está o estado inicial do sistema. Quando consideram-se doses crescentes de cobre adicionadas ao solo, o valor da energia livre pode revelar a magnitude da força de reação entre o metal e a superfície do adsorvente. Quanto maior seu valor, maior será a força de determinada reação (Alleoni \& Camargo, 1998). Dentro desse contexto, o objetivo desse trabalho foi estudar a energia livre da reação de adsorção de cobre em três solos do Estado de São Paulo.

\section{MATERIAL E MÉTODOS}

Foram utilizadas amostras de terra do horizonte superficial $(0-0,2 \mathrm{~m})$ e subsuperficial (na maior expressão do horizonte de diagnóstico) de três solos do Estado de São Paulo : 1) Terra Roxa Estruturada (TE), textura muito argilosa, município de Ribeirão Preto-SP; 2) Latossolo Roxo (LR) ácrico, textura muito argilosa, localizado no município de Ribeirão Preto-SP; 3) Latossolo variação Una (LU) ácrico, textura média, localizado em Guaíra-SP.

Os Latossolos são oxídicos, muito intemperizados, enquanto a Terra Roxa Estruturada apresenta estádio menos avançado de intemperismo, servindo assim como comparação por apresentar carga líquida negativa ao longo de todo o perfil. Os Latossolos escolhidos apresentam texturas contrastantes, para caracterizar a adsorção do metal em materiais com diferentes conteúdos de argila, uma vez que este atributo físico é um dos mais importantes em estudos de fenômenos de superfície que ocorrem nos colóides do solo. Alguns dos atributos químicos, físicos e mineralógicos dos solos estudados estão nas TABELAS 1 e 2.

Os solos foram caracterizados conforme metodologia preconizada por Camargo et al. (1986), sendo analisados os seguintes atributos: carbono orgânico pela oxidação da matéria orgânica com solução sulfo-crômica; pH em água e $\mathrm{KCl} 1 \mathrm{~mol} \mathrm{~L}^{-1}$, utilizando-se relação solo-solução 
TABELA 1 - Atributos químicos e mineralógicos dos solos estudados.

\begin{tabular}{|c|c|c|c|c|c|c|c|c|c|c|c|c|}
\hline Solo & Prof. & $\mathrm{pH}$ & $\Delta \mathrm{pH}$ & C & $\mathrm{H}+\mathrm{Al}$ & Al & SB & $\mathrm{CTC}_{\mathrm{t}}$ & $\mathrm{RC}$ & PESN & Caulinita & Gibsita \\
\hline & $\mathrm{m}$ & $\mathrm{H}_{2} \mathrm{O}$ & & $\mathrm{g} \mathrm{kg}^{-1}$ & 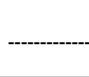 & $\mathrm{mm}$ & olc kg & 1 & $\begin{array}{l}\text { mmolc/ } \\
\mathrm{kg} \text { arg. }\end{array}$ & & g kg & $g^{-1}$ \\
\hline \multirow[t]{2}{*}{ TE } & $0-0,2$ & 5,7 & $-0,9$ & 26 & 59 & 2 & 106 & 166,8 & 131 & 3,7 & 249 & 59 \\
\hline & $0,7-0,9$ & 6,0 & $-0,7$ & 7 & 27 & 0 & 54 & 80,8 & 77 & 3,6 & 263 & 80 \\
\hline \multirow[t]{2}{*}{ LR } & $0-0,2$ & 6,4 & $-0,9$ & 20 & 30 & 0 & 63 & 92,9 & 105 & 3,6 & 172 & 375 \\
\hline & $1,0-1,4$ & 5,3 & $+0,3$ & 5 & 14 & 0 & 8 & 22,2 & 13 & 5,7 & 216 & 431 \\
\hline \multirow[t]{2}{*}{ LU } & $0-0,2$ & 4,6 & $-0,5$ & 16 & 36 & 6 & 20 & 43,4 & 38 & 3,4 & 248 & 200 \\
\hline & $1,0-1,3$ & 5,1 & $+0,6$ & 6 & 11 & 0 & 9 & 14,0 & 7 & 6,1 & 196 & 189 \\
\hline
\end{tabular}

$\mathrm{TE}=$ Terra Roxa Estruturada; $\mathrm{LR}=$ Latossolo Roxo; $\mathrm{LU}=$ Latossolo variação Una; $\mathrm{SB}=$ soma de bases; $\mathrm{CTC}_{\mathrm{t}}=\mathrm{CTC}$ total; $\mathrm{RC}=$ retenção de cátions; $\mathrm{PESN}=$ ponto de efeito salino nulo.

TABELA 2 - Teores de óxidos (totais, livre e mal cristalizados), índice ki, argila e SE.

\begin{tabular}{|c|c|c|c|c|c|c|c|c|c|c|}
\hline Solo & Prof. & $\mathrm{Fe}_{\mathrm{t}}$ & $\mathrm{Fe}_{\mathrm{d}}$ & $\mathrm{Fe}_{\circ}$ & $\mathrm{Al}_{\mathrm{t}}$ & $\mathrm{Al}_{\mathrm{d}}$ & $\mathrm{Al}_{0}$ & ki & Argila & SE \\
\hline & $\mathrm{m}$ & ---- & --י--י- & . & g & -1 & & 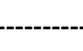 & ----- & $\mathrm{m}^{2} \mathrm{~g}^{-1}$ \\
\hline \multirow[t]{2}{*}{ TE } & $0-0,2$ & 270 & 150 & 12 & 167 & 23 & 8 & 1,89 & 820 & 134 \\
\hline & $0,7-0,9$ & 284 & 150 & 14 & 195 & 21 & 7 & 1,74 & 700 & 151 \\
\hline \multirow[t]{2}{*}{ LR } & $0-0,2$ & 320 & 164 & 13 & 195 & 26 & 10 & 0,93 & 600 & 65 \\
\hline & $1,0-1,4$ & 341 & 185 & 9 & 195 & 21 & 7 & 0,87 & 645 & 76 \\
\hline \multirow[t]{2}{*}{ LU } & $0-0,2$ & 66 & 39 & 2 & 91 & 22 & 6 & 0,98 & 350 & 65 \\
\hline & $1,0-1,3$ & 96 & 43 & 2 & 125 & 31 & 8 & 0,97 & 450 & 53 \\
\hline
\end{tabular}

$\mathrm{Fe}_{\mathrm{t}}=$ óxido de ferro total; $\mathrm{Fe}_{\mathrm{d}}=$ óxido de ferro "livre"; $\mathrm{Fe}_{\mathrm{o}}=$ óxido de ferro mal cristalizado;

Alt = óxido de alumínio total; Ald = óxido de alumínio "livre"; Alo = óxido de alumínio mal cristalizado; índice $\mathrm{ki}=\left(\% \mathrm{SiO}_{2} / 60\right) /\left(\mathrm{Al}_{2} \mathrm{O}_{3} / 102\right) ; \mathrm{SE}=$ superfície específica

1:2,5; cálcio, magnésio, potássio e sódio com extração por resina trocadora de íons e posterior leitura no espectrofotômetro de absorção atômica ( $\mathrm{Ca}$ e $\mathrm{Mg}$ ) e fotômetro de chama ( $\mathrm{K}$ e $\mathrm{Na}$ ); teores totais de óxidos (Al, $\mathrm{Fe}$ e $\mathrm{Si}$ ): obtidos após ataque sulfúrico; ferro e alumínio livres: obtidos em meio tamponado com bicarbonato de sódio, reduzindose o ferro com ditionito de sódio; ferro e alumínio mal cristalizados ou microcristalinos: obtidos por solubilização de ferro e alumínio amorfos em ácido oxálico e seu sal de amônio; ponto de efeito salino nulo (PESN): determinado pelo método de titulação com $\mathrm{H}^{+}$e $\mathrm{OH}^{-}$a três diferentes concentrações de $\mathrm{KCl}$, sendo o PESN o valor do $\mathrm{pH}$ no ponto de intersecção das curvas de titulação; superfície específica (SE): método de Heilman et al. (1965), baseado na retenção do éter monoetílico do etilenoglicol (EMEG), com as modificações propostas por Cihacek \& Bremner (1979) e Ratner-Zomar et al. (1983); análise granulométrica pelo método do densímetro; análise mineralógica quantitativa com auixílio de análise térmica diferencial (ATD).

Após a coleta, os solos foram secos ao ar e, posteriormente, peneirados em peneira com abertura de malha de dois milímetros. Pesou-se 
o correspondente a $2,00 \mathrm{~g}$ de terra fina seca em estufa e adicionaram-se $20 \mathrm{ml}$ de solução $0,0025 \mathrm{~mol} \mathrm{~L}^{-1}$ de $\mathrm{Ca}\left(\mathrm{NO}_{3}\right)_{2}$ contendo $\mathrm{Cu}\left(\mathrm{NO}_{3}\right)_{2}$ nas concentrações de $0,5,10,20,50,100,200$, 300,400 e $600 \mathrm{mg} \mathrm{L}^{-1}$ de cobre. Utilizou-se nitrato como suporte devido à menor habilidade em complexar cátions metálicos (Guilherme \& Anderson, 1998). As determinações foram realizadas em triplicata.

A partir dos resultados do experimento de adsorção de cobre, foram calculadas os respectivos valores de energia livre das reações por meio da equação : $\triangle \mathrm{G}_{0}=\mathrm{RT}$ (In cobre solução de equilíbrio - In cobre adicionado), sendo $\Delta \mathbf{G}_{0}=$ energia livre $\left(\mathrm{J} \mathrm{mol}^{-1}\right), \mathrm{R}=$ constante universal dos gases $\left(8,30 \mathrm{~J} \mathrm{~mol}^{-1} \mathrm{~K}^{-1}\right)$ e $\mathrm{T}=298 \mathrm{~K}$.

\section{RESULTADOS E DISCUSSÃO}

Os resultados de energia livre, em módulo, estão expressos na Figura 1. Observase que com o aumento na concentração de cobre na solução houve decréscimo na energia livre da reação de adsorção, indicando que quanto mais concentrada a solução, menos fortemente o elemento foi retido. Esses resultados concordam com as informações de literatura, segundo as quais o cobre, em baixas concentrações, forma ligações de alta estabilidade com a superfície dos colóides (Sparks, 1995). Porém, à medida que os sítios de adsorção vão se saturando, diminui a afinidade do solo pelo metal (Petruzzelli et al., 1985)

TE

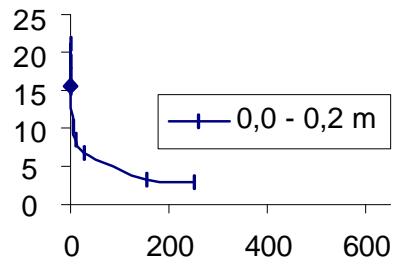

Cu em equilíbrio $\left(\mathrm{mg} \mathrm{L}^{-1}\right)$

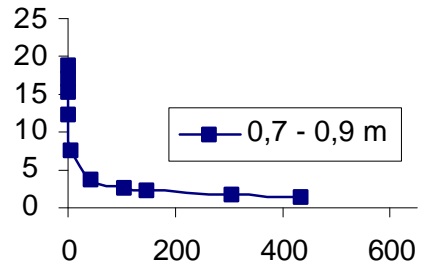

$\mathrm{Cu}$ em equilíbrio $\left(\mathrm{mg} \mathrm{L}^{-1}\right)$

LR

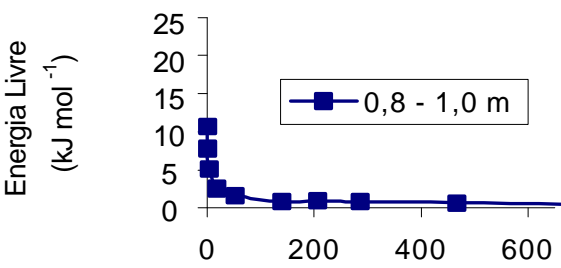

$\mathrm{Cu}$ em equilíbrio $\left(\mathrm{mg} \mathrm{L}^{-1}\right)$
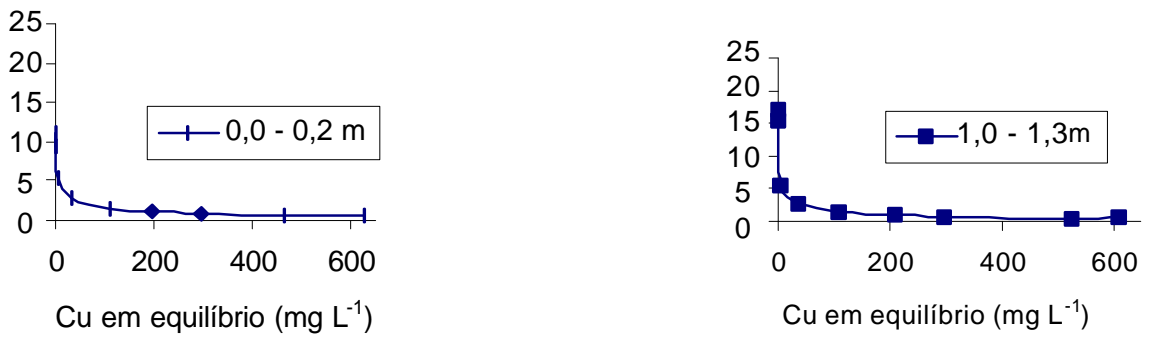

Figura 1 - Valores de energia livre (em módulo) das reações de adsorção de cobre em camadas superficiais e subsuperficiais de três solos (TE = Terra Roxa Estruturada, LR = Latossolo Roxo e $\mathrm{LU}=$ Latossolo variação Una). 
Para todas as concentrações de cobre e nos três solos estudados, o sinal de $\Delta G_{0}$ foi negativo, mostrando que as reações foram termodinamicamente espontâneas. Resultados semelhantes foram obtidos para adsorção de boro em solos tropicais por Alleoni \& Camargo (1998), que observaram valores negativos de energia livre em todas as concentrações de boro estudadas.

Os valores médios de energia livre foram superiores no horizonte superficial, possivelmente, devido ao maior teor de matéria orgânica, haja vista a acentuada afinidade do cobre pela mesma (McLaren \& Crawford, 1973; Bibak, 1994). O alto grau de seletividade mostrado entre o cobre e a matéria orgânica nas condições do experimento, é, provavelmtente, devido à formação de complexos de esfera interna, também referidos como adsorção específica (Guilherme \& Anderson, 1998). Além disso, o balanço de cargas negativo, no horizonte superficial, proporcionou maior adsorção do metal, em comparação ao horizonte subsuperficial, que apresentou balanço positivo de cargas. Nos horizontes subsuperficiais, nos quais os teores de matéria orgânica foram menores (TABELA 1), houve correspondente redução nos valores de energia livre. Porém, no horizonte subsuperficial do LU, devido à predominância da fração mineral, principalmente gibsita, a afinidade do complexo de troca pode ter sido superior, a baixas concentrações do elemento, do que no horizonte superficial (TABELA 1). Resultados semelhantes foram obtidos por Bibak (1994) que, estudando óxidos de ferro e alumínio sintéticos, observou que o óxido de alumínio foi capaz de reter maiores quantidades de cobre, devido aos mecanismos de adsorção específica. Apesar dos conteúdos de matéria orgânica nos horizontes subsuperficiais dos solos estudados serem semelhantes, a TE apresentou maior valor de energia livre em relação aos Latossolos, possivelmente devido aos teores de óxidos de ferro e alumínio (Figura 1). Resultados semelhantes foram obtidos por Cavallaro \& McBride (1984), que concluíram que em solos com baixos conteúdos de matéria orgânica, os óxidos de ferro e alumínio passam a ter maior efeito na adsorção de cobre do que a matéria orgânica, especialmente nos horizontes subsuperficiais. Entre os Latossolos, em subsuperfície, o LU apresentou maior energia livre, provavelmente devido ao efeito do alumínio livre (TABELA 2)
(Msaky \& Calvet, 1990). Não apenas o conteúdo os óxidos é importante, mas também sua natureza e o grau de cristalinidade. No LU o alumínio livre representa $25 \%$ do total, enquanto no LR corresponde a $11 \%$ do total.

Considerando ambos os horizontes, em média, a TE apresentou maior energia livre em relação ao $L R$ e $L U$, devido principalmente à sua textura, aos conteúdos de óxidos de ferro e alumínio, à superfície específica e ao balanço negativo de cargas ao longo de todo seu perfil. Já os Latossolos não diferiram entre si. $\mathrm{Na}$ TE houve predomínio de cargas negativas, pois os valores de $\mathrm{pH}$, em superfície e subsuperfície, foram superiores ao PESN, o que favoreceu a adsorção do cobre. Os maiores valores de superfície específica na TE em relação aos Latossolos, devido à predominância de caulinita na fração mineral, pode ter favorecido as reações de adsorção (TABELA 2). Os Latossolos apresentaram valores de PESN superiores aos da TE, devido ao seu estado mais avançado de intemperismo, expressos pelos respectivos índices ki, e a predominância de óxidos de ferro e alumínio na fração mineral, proporcionando balanço positivo de cargas. A diferença na magnitude de cargas entre os solos, expressas pelo valor do $\triangle \mathrm{pH}$ e CTC superiores na TE (TABELA 1), podem ter contribuído para a adsorção do metal e, consequentemente, para os valores de energia livre das reações. Porém, observou-se que esses valores foram expressivos, apesar do balanço positivo de cargas nos Latossolos, demonstrando que não apenas mecanismos de adsorção eletrostática estão envolvidos no fenômeno, uma vez que, nessas condições, deveria haver repulsão entre as cargas positivas da superfície dos colóides e o cobre. Para estes casos, devese realçar a existência de mecanismos de adsorção específica, relacionados principalmente com a predominância de óxidos de ferro e alumínio.

O teor de argila não parece ter influenciado significativamente os valores de energia livre ao longo do perfil, uma vez que o LU, apesar do menor contéudo de argila, apresentou valores de energia livre semelhantes ou superiores ao LR. Esses resultado estão de acordo com os encontrados por Sadiq (1981), que estudando solos com diferentes conteúdos de argila não encontrou correlação entre a adsorção de cobre e teor de argila. 


\section{CONCLUSÃO}

A energia livre de adsorção de cobre foi maior nas menores concentrações de cobre em equilíbrio e nos horizontes com maiores teores de matéria orgânica e óxidos de ferro e alumínio. Pode-se considerar que, para as concentrações de cobre estudadas, as reações de adsorção são espontâneas.

\section{AGRADECIMENTOS}

À FAPESP e à CAPES, pela concessão da bolsa de mestrado à primeira autora.

\section{REFERÊNCIAS BIBLIOGRÁFICAS}

ALLEONI, L.R.F.; CAMARGO, O.A. Energia livre das reações de adsorção de boro em solos. Revista de Agricultura, v.73, p.319-330, 1998.

BIBAK, A. Cobalt, copper and manganese adsorption by aluminium and iron oxides and humic acid. Communications in Soil Science and Plant Analysis, v.25, p.3229-3239, 1994.

CAMARGO, O.A.; MONIZ, A.C.; JORGE, J.A.; VALADARES, J.M.A.S. Métodos de análise química, mineralógica e física de solos do IAC. Campinas: IAC, 1986. 94p.

CAVALLARO, N.; McBRIDE, M.B. Zinc and copper sorption and fixation by an acid soil clay: effect of selective dissolution. Soil Science Society of America Journal, v.48, p.1050-1054, 1984.

CIHACEK, J.L.; BREMNER, J.M. A simplified ethyleneglycolmonoethyl procedure for assessment of soil surface area. Soil Science Society of America Journal, v.43, p.821-822, 1979.

FAO. Soil map of the world. Wageningem: ISRIC, 1989. 138p. (FAO.UNESCO Technical Paper, 20).

GUILHERME, L.R.G.; ANDERSON, S.J. Copper sorption kinetics and sorption hysteresis in two oxide-rich soils (Oxisols). In: VENNE, E.A. (Ed.) Adsorption of metals by geomedia : variables, mechanisms and model applications. San Diego: Academic Press, 1998. cap.9, p.209-228.

HEILMAN, M.D.; CARTER, D.L.; GONZALES, C.L. The ethylene glycol monoethyl ether technique for determining soil surface area. Soil Science, v.100, p.409-413, 1965.

MATTIGOD, S.V.; PAGE, A.L. Assessment of metal pollution in soil. In: THORNTON, I. (Ed.) Applied Environmental geochemistry. London: Academic Press, 1983. cap12, p.355-394.
McBRIDE, M.; SAUVÉ, S.; HENDERSHOT, W. Solubility control of $\mathrm{Cu}, \mathrm{Zn}, \mathrm{Cd}$ e $\mathrm{Pb}$ in contamined soils. European Journal of Soil Science, v.48, p.337-346, 1997.

McLAREN, R.G.; CRAWFORD, D.V. Studies on soil copper: II. The specific adsorption of copper by soils. Journal of Soil Science, v.24, p.443-452, 1973.

MSAKY, J.J.; CALVET, R. Adsorption behavior of copper and zinc in soils: influence of $\mathrm{pH}$ on adsorptions characteristics. Soil Science, v.150, p.513-522, 1990.

PETRONIO, B.M.; D'ORAZIO,D.; PETRUZZELLI, G.; GENNARO, M.C.; VANNI, A.; LIBERATORI, A. Characterization of the liquid phase of sewage sludge. Effects on copper (II) adsorption by soil. Environmental Technology, v.17, p.439-444, 1996.

PETRUZZELLI, G.; GUIDI, G.; LUBRANO, L. Ionic strength effect on heavy metal adsorption by soil. Communications in Soil Science and Plant Analysis, v.16, p.971-986, 1985.

PEZZAROSSA, B.; MALORGIO, F.; LUBRANO, L.; TOGNONI, F.; PETRUZZELLI, G. Phosphate fertilizer as a source of heavy metals in protected cultivation. Communications in Soil Science and Plant Analysis, v.21, p.737-751, 1990.

RATNER-ZOMAR, Y.; BANIN, A.; CHEN, Y. Oven drying as a pretreatment for surface area determinations of soils and clays. Soil Science Society of America Journal, v.47, p.1056-1058, 1983.

SADIQ, M. The adsorption characteristics of soil and sorption of copper, manganese e zinc. Communications in Soil Science and Plant Analysis, v.12, p.631-642, 1981.

SALAM, A.K.; HELMKE, P.A. The pH dependence of free ionic activities and total dissolved concentrations of copper and cadmium in soil solution. Geoderma, v.83, p.281-291, 1998.

SINGH, M. Retention of added copper by two soils as affected by organic matter, $\mathrm{CaCO}_{3}$ and exchangeable ions. Geoderma, v.5, p.219-227, 1971.

SPARKS, D.L. Environmental soil chemistry. San Diego: California, 1995. cap.5, p.99-139: Sorption phenomena on soils.

Recebido para publicação em 28.05.99

Aceito para publicação em 03.09.99 